\title{
Do you have Standard Membership Protocols?
}

\section{By Gary Nelson}

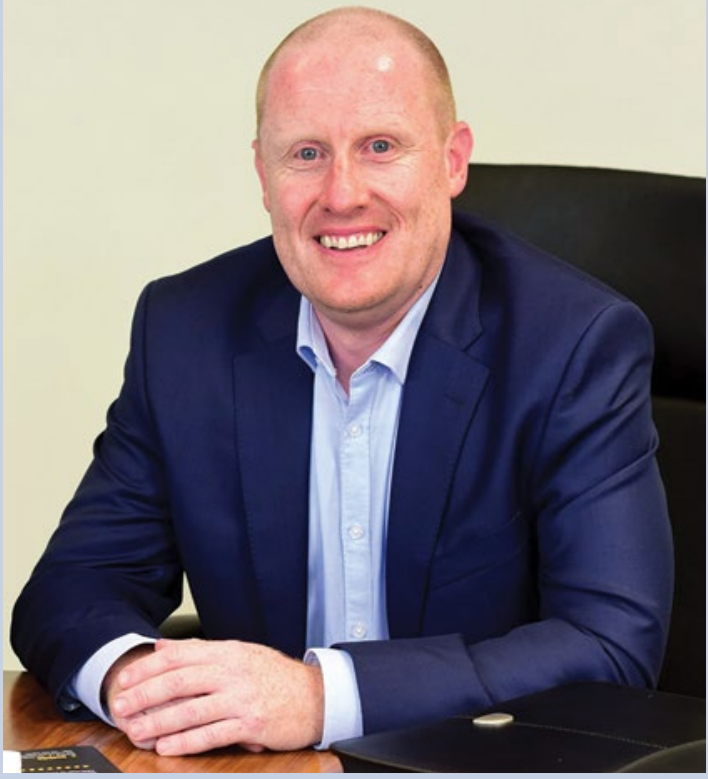

We've all heard about Standard Membership Protocols (SOPs), especially in the run-up to reopening practices after lockdown. But have you stopped to consider SMPs?

That's your Standard Membership Protocols - SMPs.

Now is the perfect time to think about introducing them into your practice, if you don't have any already.

\section{A plan for your future}

Practice Plan's team of experienced professionals has supported over 1,500 dental practices to transform the profitability of their business through the combination of a well-populated plan and personalised support including marketing, events and training.

Our Regional Support Managers are on hand to provide business advice that will not only help you grow your membership, but help all elements of your practice flourish. Gary Nelson, Area Support Manager for Northern Ireland, shares his ideas for boosting your plan membership uptake.

to implement change and help crisis-proof your business in the future?

Putting in place some SMPs can help you to do this, and they should be applicable to the whole team. I wanted to share my observations, based on 20 years of helping develop large membership-based practices across the country.

\section{Leadership is key}

If the leader of the business is passionate and assertive with patients that the best preventive approach and healthcare package for them is to become a member, the patients generally join the plan.

\section{You had me at hello}

You rarely get a second chance to make a first impression. The largest membership plan practices all mention membership at reception prior to entering the surgery.

\section{'A well-populated plan has proved to be a good foundation for navigating one of the toughest times ever faced by the dental profession'}

Throughout lockdown, patients on a plan proved to be incredibly loyal in continuing to pay their monthly fee - only a minimal amount of Practice Plan patients cancelled their plan in the early weeks after practices closed their doors. As a result, practices with a well-populated plan fared well during lockdown as it helped to mitigate the hardship felt by many others.

As we are now on the run up to the New Year, I ask you, what better time is there
Note the word 'mention'. All too often it's left solely to the reception team to 'sell' membership, but patients want to hear it from the clinician, and in many of the large membership-based practices, the clinician communicates it best.

\section{The team must believe}

When did you last have a long chat about membership with the team? By which I mean, have an internal team session, iron out any problems, talk about what works, what used to work, why we stopped doing that, what could we do, etc. The best ideas are those that you believe in, together as a team.

\section{People buy from people}

You can have the best designed brochure and point-of-sale material in the world, but people buy from people. Marketing literature is there to back up the message. Keep it simple, believe in it and remember $80 \%$ of any face-to-face communication will always be told by your body language and tone of voice. If you believe in it, the patient can tell!

\section{The handover is the key}

The final piece of the jigsaw: after you've discussed the membership plan with the patient, walk them back to reception. It is a lovely caring approach and it's a way of explaining to your reception team that $\mathrm{Mr}$ Jones needs to get signed up as a member.

If you do not ask, the answer will always be no - it's something that many people shy away from in fear of rejection. But it's a fact of life: if you don't ask you will never progress.

A well-populated plan has proved to be a good foundation for navigating one of the toughest times ever faced by the dental profession. The ideas above can help you to grow your plan and provide you with that extra security in both good times, and bad.

\section{About Gary Nelson}

Gary Nelson is an Area Manager with Practice Plan and has successfully run his own business for ten years prior to joining the team. 